\title{
Coordinated Nano-Scale EDS and EELS Measurements of Lunar Space-Weathered Material
}

\author{
K. D. Burgess ${ }^{1}$ and R. M. Stroud ${ }^{1}$
}

${ }^{1}$ Materials Science and Technology Division, U.S. Naval Research Laboratory, Washington, DC, USA

Investigations of samples from the Moon brought back by the Apollo astronauts have revealed that the vast majority of the tiny rock and mineral fragments that make up much of lunar regolith are coated with thin rims of altered material, on the order of a couple hundred nanometers or less [1]. Although this altered, or space weathered, material was observed soon after return of the samples [2], it was not for another 20 years that detailed measurements were able to reveal the compositional and nano-structural complexity of individual rims [3]. The continuing development of solid-state energy dispersive X-ray spectrometers (EDS), first reported on just 18 months before return of the first lunar samples [4], and integration of the technique with transmission electron microscopy (TEM), was a key step in this advancement of our understanding of lunar samples. Our current ability to quickly acquire EDS image cubes at very high spatial resolution, and coordinate that data with electron energy loss spectroscopy (EELS) and detailed imaging provides a powerful tool for revealing new secrets of these valuable samples.

Space weathered rims on many lunar grains are highly enriched in Fe, and much of that Fe is present in inclusions known as nanophase metallic iron $\left(\mathrm{npFe}^{0}\right)$. Recent work has used EELS to demonstrate that some proportion of the $n \mathrm{FFe}^{0}$ inclusions are in fact oxidized [5]. By combining EELS and EDS data, we can examine the relationship between where that oxidation has taken place and the composition of the surrounding altered material or underlying unaltered substrate, which can provide additional information about processes occurring on the lunar surface. We used focused ion beam (FIB) microscopy to prepare sections from several lunar soil grains, and examined them using a NION UltraSTEM-X at $200 \mathrm{kV}$ equipped with a Gatan Enfinium ER EEL spectrometer with and a Bruker SDD-EDS detector.

A FIB section from a plagioclase grain from soil 79221 shows a variety of space weathering features, including a region with five distinct layers deposited on a glassy substrate (Fig. 1). The layers range in thickness from $\sim 2 \mathrm{~nm}$ to $18 \mathrm{~nm}$ and are highly variable in composition, oxidation state, and areal density of Fe-rich inclusions. The boundaries between them are sharp perpendicular to the grain surface. There is no obvious relationship between degree of oxidation and major element composition in each layer. However, across all samples, oxidized material was more common on grains of glass or plagioclase, which are poor in Fe compared to olivine and pyroxene.

Oxidized material in lunar samples has not been reliably identified using other techniques such as Mössbauer [6]. By knowing substrate and rim composition, we can calculate the maximum amount of oxidized material in the soils that could be present while remaining below detection limits. For oxidized rims as in Fig. 1, assuming each of the layers present here has the same lateral extent and consistent thickness, $\mathrm{Fe}^{3+}$ makes up $18 \pm 7$ at $\%$ of the total $\mathrm{Fe}$ in this space weathered region. The bulk glass substrate here has a significant number of $\mathrm{nFe}^{0}$ inclusions and an average of 5 at $\% \mathrm{Fe}$, most of which is in the inclusions. The oxidized material would be undetectable in a plagioclase grain 40-50 $\mu \mathrm{m}$ across or an olivine or pyroxene grain larger than $\sim 5 \mu \mathrm{m}$, sizes well within the range of that seen in lunar soils.

The application of advanced electron microscopy techniques to lunar materials has allowed us to observe 
and analyze space weathering features at an unprecedented scale. Such data provide a necessary perspective for recognizing processes that operate in nature at the nanoscale and yet influence questions as large as our understanding of the formation and evolution of the Moon.

\section{References:}

[1] Keller, L.P., and D.S. McKay (1997) GCA, 61, 2331.

[2] Bibring, J.P., et al. (1972) Science, 175, 753.

[3] Keller, L.P., and D.S. McKay (1993) Science, 261, 1305.

[4] Fitzgerald, R., et al. (1968) Science, 159, 528.

[5] Thompson, M.S., et al. (2016) M\&PS, 51, 1082.

[6] Housley, R.M., et al. (1972) LPSC, 3, 1065.

[7] The authors acknowledge funding from the RIS ${ }^{4}$ E node of NASA's Solar System Exploration Research Virtual Institute.
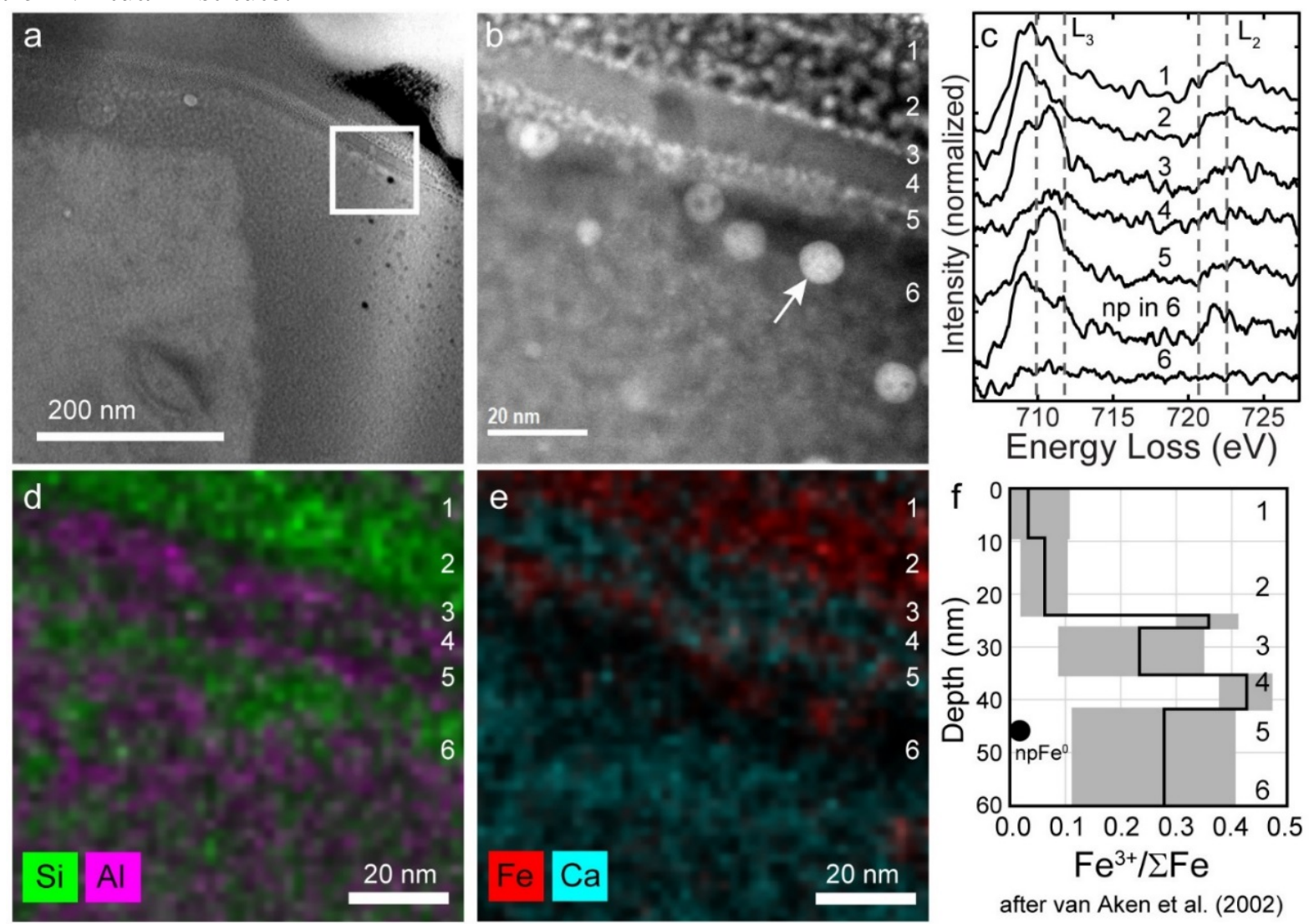

Figure 1. (a) BF TEM image of a lunar soil grain, showing the amorphous coating on the plagioclase on the left with npFe-rich glass to the right. The vapor deposited layers are continuous across the substrate phases but show significant variation in composition and oxidation state between layers. (b) HAADF image from the region highlighted in (a). At least five distinct layers with varying major element composition are present in the vapor deposited rim coating the silicate glass substrate. A few of the larger $\mathrm{npFe} \mathrm{P}^{0}$ have a pitted appearance. (c) EELS data from layers identified in (b) showing variations in oxidation state and Fe content. Two of the layers contain very small $(1-3 \mathrm{~nm}) \mathrm{npFe}^{\mathrm{x}}$ inclusions that are highly oxidized (layers 3 \& 5), while the middle layer is poor in Fe and has no inclusions (layer 4). The arrow in (b) points to the inclusion from which the spectrum was taken. (d-e) EDS element maps of the same region show compositional differences in addition to Fe oxidation state. (f) The oxidation state for each layer is calculated from the modified white line intensity ratio of van Aken et al. (2002) (dotted lines in (c)). 